\title{
Holmium Laser Enucleation of the Prostate
}

\author{
Marcelino E. Rivera, MD, James E. Lingeman, MD, and Amy E. Krambeck, MD
}

\begin{abstract}
Introduction: Holmium laser enucleation of the prostate (HoLEP) offers superior voiding outcomes to traditional transurethral resection and less morbidity than open simple prostatectomy. Likewise, HoLEP has been determined to result in excellent outcomes regardless of gland size. We present a step-by-step surgical approach to HoLEP describing both the traditional enucleation technique and a modified "top-down" surgical technique.

Materials and Methods: In this video, two techniques are presented that were performed by two (A.E.K., J.E.L.) surgeons at our institution.

Results: In the examples of the two enucleation techniques mentioned, outcomes are similar with regard to surgical and functional outcomes.

Conclusions: HoLEP as a treatment for BPH with associated lower urinary tract symptoms (LUTS) results in excellent patient outcomes and can be offered to patients regardless of prostate volume.
\end{abstract}

Keywords: bladder, benign prostatic hyperplasia, Holmium Laser Enucleation of the Prostate

\section{Indications}

$\mathbf{P}$ ATIENTS WHO ARE CANDIDATES for any bladder outlet procedure because of benign prostatic hyperplasia (BPH) can be selected for holmium laser enucleation of the prostate (HoLEP) (see Supplementary Video S1; Supplementary Data are available online at www.liebertpub.com/ end).

\section{Preoperative Preparation}

Although not required, uroflowmetry and/or pressure flow urodynamics can be utilized to assess degree of obstruction and detrusor function. If concerns exist for urethral stricture disease, in-office cystoscopy can likewise be performed. Prostate sizing, in the form of transrectal ultrasonography, $\mathrm{CT}$, or MRI, is utilized to predict procedure duration and operating room utilization. It is preferable to discontinue all anticoagulant therapy; however, this is not required and HoLEP can be performed safely with patients who are anticoagulated. A preoperative urine culture is obtained and tailored antibiotic therapy is selected.

\section{Patient Positioning}

Patients are positioned in the dorsal lithotomy position with lower extremity pressure points padded to avoid potential neuropraxia. Sequential compression devices are placed for venous thromboembolism prophylaxis.

\section{Surgical Steps}

(1) The urethra is calibrated utilizing Van Buren sounds to $30-32 \mathrm{~F}$ and, if necessary, an Otis urethrotome can be utilized to incise the distal penile urethra and meatus.

(2) The laser endoscope (Fig. 1), which contains an offset $30^{\circ}$ lens, and a modified inner sheath to accommodate a laser fiber guide, is deployed into the bladder through a Timberlake obturator in a $28 \mathrm{~F}$ continuous flow sheath.

(3) Initial cystoscopy is performed to survey the bladder, define prostate anatomic configuration (bilobar vs trilobar), the distal extent of the dissection, and the ureteral orificies.

(4) During traditional HoLEP, in the setting of a median lobe, incisions are made at the 5 and 7 o'clock positions and carried down to the surgical capsule. To enucleate the median lobe, the urothelium is transected at the level of the verumontanum and the lobe is enucleated into the bladder.

- With the "top-down" approach and with a bilobar prostate configuration, a single incision is made at the bladder neck and to the depth of the surgical capsule. Laser settings during this portion of the dissection are $2 \mathrm{~J}$ and $40 \mathrm{~Hz}$ (Fig. 2).

(5) During the conventional approach, apical dissection is performed by incising the urothelium overlying the right or left lobe. Laser settings during this portion of the procedure are $2 \mathrm{~J}$ and $20 \mathrm{~Hz}$.

- However, with the top-down approach, an incision is made to divide the anterior commissure and

Indiana University Department of Urology, Indianapolis, Indiana. 


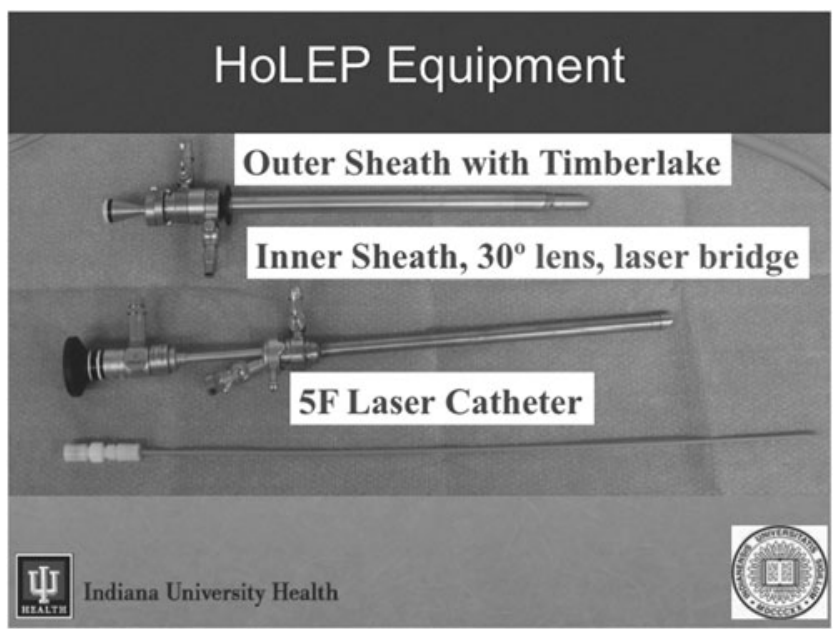

FIG. 1. Laser endoscope setup.

dissection is performed to establish the anterior plane between adenoma and capsule (Fig. 3).

(6) Once the adenoma is dissected anteriorly in the traditional approach, the bladder neck fibers are divided in the midline the bladder is entered (Fig. 4).

- During the "top-down" approach, once the anterior commissure is divided and the plane between capsule and adenoma is established on both lobes, the bladder neck is incised.

(7) The true lumen is entered, the endoscope is inverted, and the anterior commissure is divided in the traditional approach (Fig. 5).
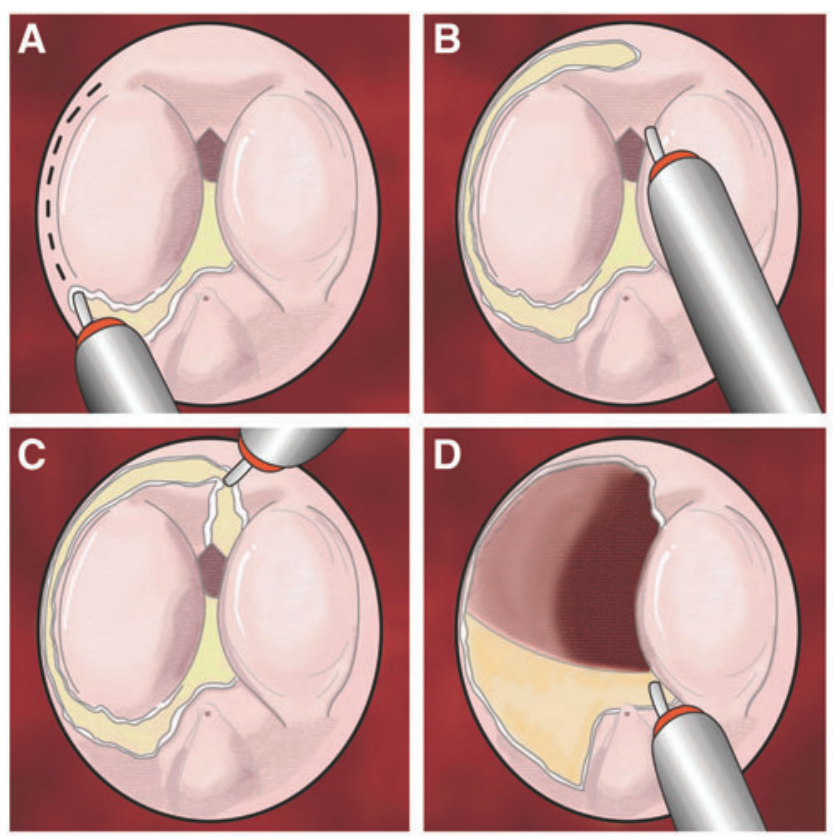

FIG. 2. Traditional bilobar enucleation, apical dissection. (A). Dissection under the lateral lobe starts lateral to the veru just proximal to the external sphincter. (B). The dissection plan is carried laterally and anteriorly past the midline. (C). The anterior commissure is then divided exposing the anteriorly dissected space. (D). The remaining attachments of the lateral lobe are severed to complete the enucleation.

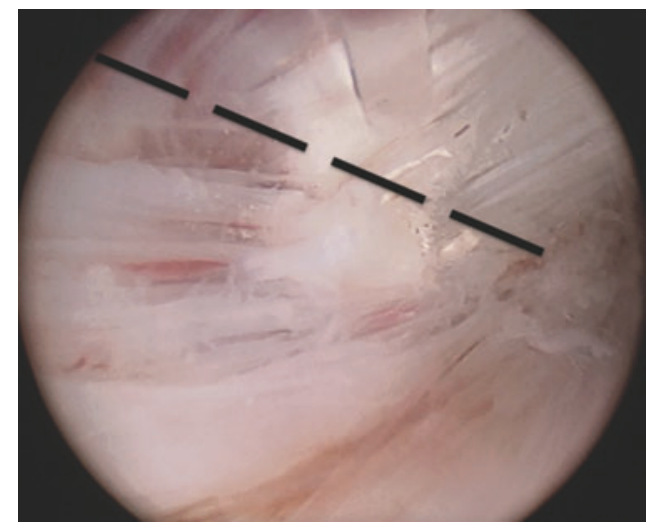

FIG. 3. "Top-down" approach, plane between capsule and adenoma identified (black lines).

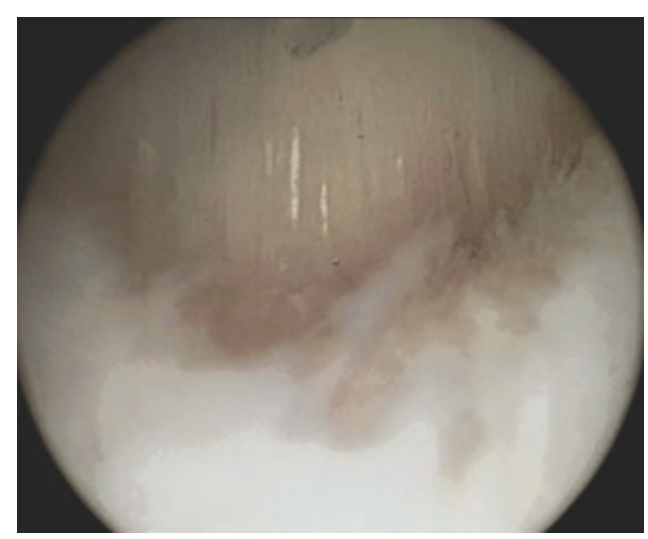

FIG. 4. Traditional approach, incising bladder neck fibers. Please note the vertical orientation of the bladder neck fibers.

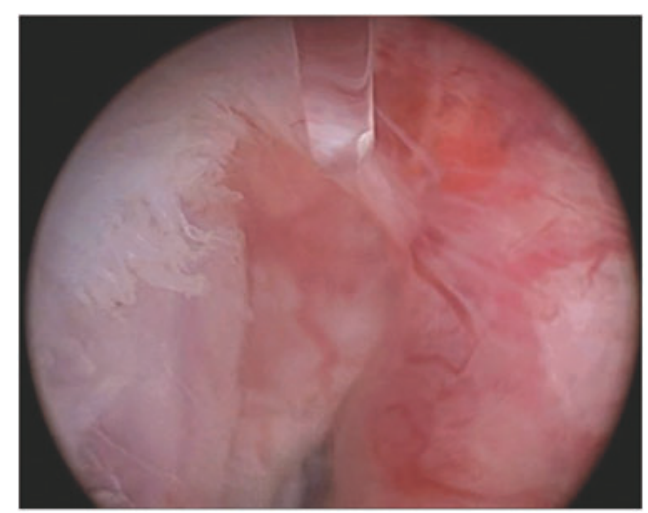

FIG. 5. Dividing the anterior commissure.

(8) To divide the mucosal strip in the traditional HoLEP, the surgeon inverts the endoscope at the level of the mid-prostate and in a circular motion, sweeps along the plane between the lateral lobe and capsule while moving distally to ensnare the mucosal strip. The external sphincter is in proximity lateral to the mucosal strip and, therefore, care must be taken to incise the mucosa in the midline. For this portion of the procedure, laser settings of $2 \mathrm{~J}$ and $20 \mathrm{~Hz}$ are utilized (Fig. 6). 


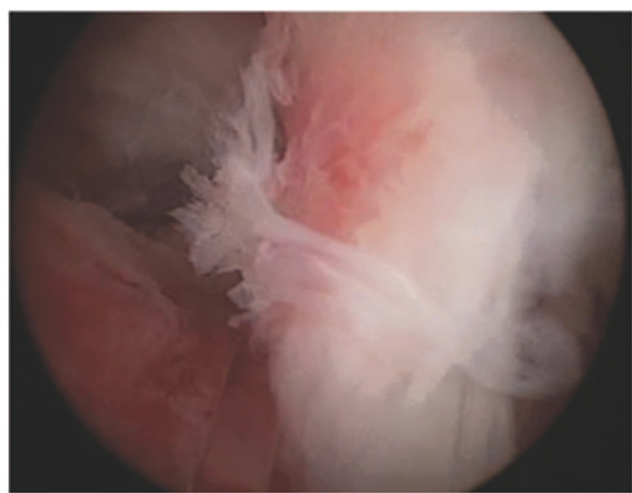

FIG. 6. Incision of the mucosal strip.

- The "top-down" approach does not require the use of the encircle technique and encounters the mucosal strip during the distal, apical extent of the lateral lobe dissection.

(9) Once the mucosal strip is divided, enculeation can be performed from lateral to medial at settings of $2 \mathrm{~J}$ and $40 \mathrm{~Hz}$.

(10) Once all tissue has been enucleated, before morcellation, the laser is defocused onto bleeding vessels to ensure hemostasis and allow for excellent visibility during morcellation.

(11) Morcellation is performed by utilizing an extended length nephroscope and the Wolf Pirhana Morcellator. Morcellator settings are typically 1500 oscillations/ min. If round denser "beach ball" tissue is encountered, settings of 1000 oscillations/min are utilized.

(12) Inspection of the capsule and bladder is performed at the conclusion of morcellation to ensure that all tissue has been morcellated and to ensure hemostasis is adequate.

\section{Post-Op Care}

Patients have a $22 \mathrm{~F}$ three-way catheter placed for continuous bladder irrigation overnight. The catheter is removed early on the first postoperative day, and once patients are able to void twice, they are dismissed home. Patients are instructed to avoid vigorous activity or heavy lifting ( $>10 \mathrm{lbs}$ ) for 1 week and intercourse for 2 weeks. Straddle activities are avoided for 1 month. Anticoagulants, if held, are resumed 5 to 7 days postoperatively once gross hematuria has subsided.

\section{Troubleshooting}

\section{Perineal urethrostomy}

HoLEP can be performed in all patients with prostates of all volumes and is an excellent procedure for prostates that have undergone prior transurethral resection, laser vaporization, microwave therapy, etc. However, there are special considerations in extremely large patients or patients with an extremely large gland $(>300 \mathrm{cc})$. A temporary perineal urethrostomy can be created to reduce the amount of urethra traversed with the laser endoscope to perform the procedure. A sound is placed into the bulbar urethra to assist with identification. A vertical skin incision over the perineum $\sim 6 \mathrm{~cm}$ in length is made. The bulbar urethra is dissected and tagged with a 3-0 absorbable suture. The bulbar urethra is incised sharply onto the sound to avoid extensive urethral injury. Once the procedure is completed, the urethra is closed and a catheter left in place for a period of at least 1 week.

\section{Capsular perforation}

If an error is made early in the learning curve of HoLEP, a capsular perforation can occur along any location of the dissection plane. If this is recognized immediately and the correct plane established over the perforation, the catheter can still be removed on the first postoperative day. However, if the capsular perforation is extensive and dissection is carried out along this false plane, the urinary catheter should be left in place for a period of several days.

\section{Recommended Videos from Videourology}

1. Hussein M.I. Y, Taglialatela D, Ceresoli F, Milesi R, Del Rosso A, Vavassori I. Holmium Laser Enucleation of the Prostate, Tips and Tricks Not Just for Beginners. January 2015, 29. https://doi.org/10.1089/vid.2014. 0040

2. Placer J, Salvador C, Trilla E, Planas J, Lozano F, Ropero J, Lorente D, López MÁ, Morote J. Holmium Laser Enucleation of the Prostate. Journal of Endourology Part B, Videourology. March 2012, 26. https://doi.org/10.1089/vid.2011.0076

3. Misrai V, Cornu J, Woo HH, Gomez-Sancha F. En Bloc Enucleation of the Prostate Using a Surgical 532nm Laser (GreenLEP) Technique: Initial Results. May 2015, 29. https://doi.org/10.1089/vid.2014.0068

\section{Supplementary Videos}

Click here for Supplementary Videos: Anterior Commissure .mp4; Anterior Dissection.mp4; Hemostasis and Morcellation .mp4; HoLEP Apical Dissection.mp4; HoLEP Videourology.mp4; Initial Set-up and Incisions.mp4; Intraoperative Special Considerations.mp4; Mucosal Strip.mp4; Top Down Technique.mp4

\section{Author Disclosure Statement}

Rivera, None; Lingeman and Krambeck, Boston Scientific and Lumenis.

$$
\begin{array}{r}
\text { Address correspondence to: } \\
\text { Amy E. Krambeck, MD } \\
\text { Department of Urology } \\
\text { Indiana University Health Physicians } \\
1801 \text { Senate Blvd } \\
\text { Indianapolis, IN } 46202
\end{array}
$$

E-mail: akrambeck@iuhealth.org

$$
\begin{aligned}
& \text { Abbreviations Used } \\
\mathrm{CT} & =\text { computed tomography } \\
\mathrm{HoLEP} & =\text { holmium laser enucleation of the prostate } \\
\mathrm{MRI} & =\text { magnetic resonance imaging }
\end{aligned}
$$

